\title{
Paternity testing under the cloak of recreational genetics
}

\author{
Nathalie Moray ${ }^{1,2}$, Katherina E Pink ${ }^{3,4}$, Pascal Borry ${ }^{1,5}$ and Maarten HD Larmuseau ${ }^{\star, 5,6}$
}

Direct-to-consumer (DTC) internet companies are selling widely advertised and highly popular genetic ancestry tests to the broad public. These tests are often classified as falling within the scope of so-called 'recreational genetics', but little is known about the impact of using these services. In this study, a particular focus is whether minors (and under what conditions) should be able to participate in the use of these DTC tests. Current ancestry tests are easily able to reveal whether participants are related and can, therefore, also reveal misattributed paternity, with implications for the minors and adults involved in the testing. We analysed the publicly available privacy policies and terms of services of 43 DTC genetic ancestry companies to assess whether minors are able to participate in testing DTC genetic ancestry, and also whether and how companies ethically account for the potential of paternity inference. Our results indicated that the majority of DTC genetic ancestry testing companies do not specifically address whether minors are able to participate in testing. Furthermore, the majority of the policies and terms of services fail to mention the vulnerability of minors and family members in receiving unexpected information, in particular, in relation to (misattributed) paternity. Therefore, recreational genetics carries both the risk of unintentionally revealing misidentified paternity, and also the risk that fathers will deliberately use these services to test their children's paternity without revealing their intentions to the mother or any other third party.

European Journal of Human Genetics (2017) 25, 768-770; doi:10.1038/ejhg.2017.31; published online 8 March 2017

\section{INTRODUCTION}

Genetic ancestry testing refers to the association of a person's origin (based on his or her DNA data) with the geographic location(s) of presumed ancestors, which is inferred through comparison with contemporary populations living in these locations. ${ }^{1}$ Due to the large public interest, the direct-to-consumer (DTC) genetic ancestry testing industry is an economic growth sector. ${ }^{2,3}$ As of early 2016, there have been more than three million tests sold. ${ }^{3}$ DTC companies widely advertise a variety of genetic and genomic ancestry tests. First, multiple unlinked diploid markers on autosomal DNA (atDNA), which are inherited from both parents, have been used for assessing patterns of genetic variation in worldwide human populations. ${ }^{4}$ Second, 'lineage analysis' represents a generational narrative about one's relatives through maternal and paternal lines of descent. ${ }^{5}$ Mitochondrial DNA (mtDNA) provides information about the female-female transmitted lineage (males also inherit mtDNA from their mother but do not transmit it to their offspring), whereas the $\mathrm{Y}$ chromosome is informative about the male-to-male transmitted lineage. ${ }^{6}$

Although genetic ancestry testing is often categorised under 'recreational genetics', the increasing public interest in DTC tests has been accompanied by growing concerns about the social, ethical and scientific implications associated with ancestry testing. $.7,8$ Research in this field has covered issues such as genetic privacy and consent, ${ }^{9}$ the use of DNA data for biomedical and clinical research ${ }^{3,7}$ and the scientific validity of ancestry inferences. ${ }^{5}$ Ancestry assessment is often wrongly perceived as the clear-cut connection between an individual's DNA and his/her racial or ethnic affiliation, ${ }^{10}$ opening up societal challenges related to stigmatisation and ethnic group identification. ${ }^{2}$ Some authors have recently expressed their concerns regarding the anonymity of donors and the biological parents of adoptive children in the context of DTC genetic testing services. ${ }^{11-14}$ Given the increasing number of DTC genetic testing companies and the potential impact of test results on individuals, families and third parties, the American Society of Human Genetics (ASHG) has recommended independent consideration of the ethical implications of DTC genetic ancestry testing. ${ }^{15}$

Considering the increasing attention being paid to the impact of DTC genetic ancestry testing and to the implications of genetic testing of minors in general, ${ }^{16}$ it is remarkable that the ability to involve minors in these specific types of 'recreational' DTC tests has not yet been addressed. Current available DTC genetic ancestry tests, which make use of atDNA and Y-chromosomal markers have the intrinsic potential to easily reveal whether participants are related., ${ }^{5,6}$ Therefore, minors are particularly vulnerable as a result of the potential for circumstances to reveal misattributed paternity, which could lead to substantial social, psychological and educational consequences for them. ${ }^{17}$ Furthermore, it will also be an emotionally distressing finding for all other parties involved, including the mother, the social father, the biological father and the close family network. ${ }^{18,19}$

Owing to these (negative) implications for minors, the research question of this study is whether and how DTC genetic ancestry testing companies account ethically for the potential use of their services for minors for (misattributed) paternity questions and paternity inferences. In addressing this question, we provide an explorative, descriptive analysis of publicly available privacy policies and terms of services for a well-defined sample of DTC genetic ancestry testing companies. The following questions guided the analysis: (i) are DTC genetic ancestry companies clear in their policies

${ }^{1}$ Centre for Biomedical Ethics and Law, KU Leuven, Leuven, Belgium; ${ }^{2}$ AZ Maria Middelares, Gent, Belgium; ${ }^{3}$ Family and Population Studies, Centre of Sociological Research, KU Leuven, Leuven, Belgium; ${ }^{4}$ Faculty of Life Sciences, Department of Anthropology, University of Vienna, Vienna, Austria; ${ }^{5}$ Leuven Institute for Genomics and Society (LIGAS), KU Leuven, Leuven, Belgium; ${ }^{6}$ Forensic Biomedical Sciences, Department of Imaging and Pathology, KU Leuven, Leuven, Belgium

${ }^{*}$ Correspondence: Dr MHD Larmuseau, Forensic Biomedical Sciences, Department of Imaging and Pathology, KU Leuven, Kapucijnenvoer 33, B-3000 Leuven, Belgium. Tel: +32 163366 63; Fax: +32 (0) 163245 75; E-mail: maarten.larmuseau@kuleuven.be

Received 10 December 2016; revised 1 February 2017; accepted 7 February 2017; published online 8 March 2017 
about whether or not minors are able to participate in testing?; (ii) to what extent do DTC genetic ancestry testing companies refer to the ethical and social considerations of handling the data of minors for paternity testing in general and paternity inference in particular?

\section{MATERIALS AND METHODS}

The companies that were included in this analysis were obtained from earlier lists published by Royal et al., ${ }^{5}$ Howard et al, ${ }^{20}$ and by the International Society of Genetic Genealogy (ISOGG hereafter; www.isogg.com, 20 January 2016). We only included companies with a working English website that are providing genetic and genomic testing services for ancestry purposes. The final list included 43 DTC companies.

The privacy policies, terms of services and the frequently asked questions (FAQ) sections of the companies' websites addressing the issue of genetic testing in minors, were analysed in January/February 2016. In addition, all information provided regarding the potential for paternity inferences and the sensitive nature of this type of information was collected.

For clustering the companies' policies and statements addressing the issues of genetic testing of minors, an adaptation of the classification of Borry et a ${ }^{21}$ was used: (i) companies that do not provide any information about testing of minors; (ii) companies that accept testing of minors; (iii) companies pointing out that their website is not aimed at minors but are not clear regarding their acceptance of DNA samples from minors; (iv) companies suggesting that their services are not meant for minors; and (v) companies with ambiguous or contradictory information regarding the participation of minors.

\section{RESULTS}

The websites of 43 DTC companies were analysed (see Supplementary Table S1). Their testing portfolios ranged from ancestry and paternity testing to genetic testing services capable of testing genetic predisposition to diseases, addictions, allergies or athletic performance $(N=11)$; predicting specific physical traits $(N=3)$; and providing evidence for adultery of one's partner based on collected items, for example, underwear $(N=6)$. Only 12 of 43 DTC companies $(27.9 \%)$ offered genetic ancestry testing services exclusively. Paternity testing services were offered by $23(53.5 \%)$ out of 43 DTC companies (see Supplementary Table S2).

The analysis showed that $20(46.5 \%)$ out of 43 companies did not provide any information about testing of minors for their genetic ancestry tests (class 1); 18 companies (41.9\%) accepted testing of minors (class 2); two companies $(4.7 \%)$ pointed out that their website was not aimed at minors but were not clear concerning the acceptance of DNA samples from minors (class 3); one company (2.3\%) implied that their services were not meant for minors (class 4); and two companies $(4.7 \%)$ were ambiguous or provided contradictory information regarding their acceptance of the participation of minors (class 5). As an illustration of the last class, the following two quotes were found on the website of one company: 'How old does a child need to be to perform a DNA test? A newborn infant can be tested. Taking a sample with a buccal swab is painless, and is not traumatic for the child (Website FAQ).' and 'We do not intentionally gather information about visitors who are under the age of 18 . If you are under the age of 18 you should not use our site or service (Privacy policy, 8. A note about children).' These quotes show that the company concurrently communicates that it accepts samples from minors (class 2) and that the website or service should only be used by adults (class 4 ).

Within the 18 companies accepting the participation of minors, 11 explicitly referred to the need for the consent of one parent or legal guardian, two companies mentioned that the customer must take local laws into account and five companies did not mention any explicit need for parental consent. However, there was no conformity among the investigated websites in regard to either the content of the information provided regarding the testing of minors or in which section of the website this information could be found.

Overall, there were no companies in the sample that specifically prohibited the use of the provided genetic ancestry testing services for paternity testing. Only 10 (23.3\%) out of 43 companies briefly referred to the notion that unexpected or sensitive results may arise from testing, although in vague and general terms. Only 3 out of these 10 companies explicitly mention the possibility of finding out that your father is not genetically your father when engaging in ancestry genetic testing.

\section{DISCUSSION}

The review of the publicly available privacy policies and terms of services of 43 DTC genetic ancestry testing companies clearly showed that information regarding the testing of minors is either absent or difficult to locate on the companies' websites. The same applies to advising the consumer of the possible risk of identifying misattributed paternity. In the first place, it is intriguing that only 18 out of 43 DTC companies $(41.9 \%)$ clearly state whether or not minors are able to participate in genetic ancestry testing. The finding that almost half of the companies in the sample do not provide information regarding their stance on testing minors is in line with previous studies in the context of DTC genetic testing for medical purposes, ${ }^{20,21}$ although the ASHG has raised this issue in the context of consent for the genetic testing of minors. Next, only three DTC genetic ancestry testing companies refer to the fact that these tests have the potential to reveal misattributed paternity and then go on to mention some ethical and social considerations in handling the data of minors for paternity testing. Therefore, the large majority of the companies pay no attention at all to the possible implications of the test results for minors, where a finding of non-paternity may apply to their parents, or for unknown individuals who may come into the picture as a result of a focused genetic ancestry database search to biologically related individuals. ${ }^{6}$ Of course, our study is limited by the very nature of looking at written company policies, which may not represent actual company practices and do not cover additional information that may be given throughout the process of providing the test kits. Nevertheless, consumers need to be sufficiently informed before they buy genetic tests, especially when the results of those tests carry the risk of negatively affecting their personal relationships and the stability of their family.

As DTC genetic ancestry testing companies do not systematically mention the potential they carry to reveal misattributed paternity, it means that they are in fact paternity tests in disguise when a minor and the attributed father (or a close family member of the father) are both being tested. Therefore, ancestry testing carries the risk of revealing misidentified paternity unexpectedly, but also the risk that fathers may intentionally use the service to secretly test their paternity. Unexpected findings of misidentified paternity are a well-recognised issue in genetic testing. ${ }^{17}$ Anderson ${ }^{22}$ reported levels around $1-2 \%$ in contemporary human populations of so-called extra-pair paternity (EPP). Based on recent genetic genealogical studies, these rates seem to be constant over the last centuries and across nations. ${ }^{23,24}$ Therefore, the detection of an unexpected misattributed paternity is always a potential outcome when related persons participate in genetic ancestry testing together. Nevertheless, consumers of DTC genetic ancestry tests have no reasonable access to appropriate support in cases where unexpected results could have a strong psychological impact. ${ }^{17}$ Therefore, participants should always be warned of the potential for the identification of misattributed paternity, especially when (highly vulnerable) minors are involved.

As consumers do not need to be explicit about their reasons for genetic ancestry testing, the implications of genetic testing of minors in 
general, and the potential for paternity inference in particular, may be underestimated. More specifically, attributed fathers may involve their children in testing to confirm their paternity if they have doubts about their biological relatedness. Along the same line, minors themselves may have doubts about their parental ancestry and seek confirmation through the use of DTC genetic ancestry testing services when another member of the family has already done the test. As none of the 43 analysed DTC genetic ancestry testing companies asked for the consent of both parents or specifically of the mother, those tests can easily be used as paternity tests in disguise without raising suspicions in the mother or third parties. This possibility of testing paternity under the name of 'recreational genetics' might be problematic in some countries where regulations regarding paternity testing recommend the mother's consent (eg, UK; http://www.bma. org.uk/advice/employment/ethics/children-and-young-people/paternitytesting-Accessed 26 November 2016) or require the consent of both parents (eg, Switzerland; http://www.ch.ch/en/paternity-test/—Accessed 26 November 2016). France is even more strict: doubtful fathers attempting to infer paternity through genetic testing without a court order or without the explicit consent of the mother risk a sentence of detention (http://www.avoiceformen.com/mens-rights/france-upholdsthe-ban-on-paternity-tests/—Accessed 26 November 2016).

Our analysis demonstrates the importance of developing an industry standard concerning the testing of minors and (misattributed) paternity as an unexpected finding in the context of DTC genetic ancestry testing. Regarding paternity testing, the following recommendations from the UK Departments of Health (UK Departments of Health 2008; BMA Ethics Department 2009) seem to be useful in the context of DTC genetic ancestry testing. First, individuals giving consent to a test should first and foremost be fully informed of its purpose and accuracy, and the potential consequences of the test result. Companies should include information, which will prompt people to consider the best interests of the child involved and the future implications for themselves and their families in commissioning a test, as part of their advertising, marketing and kit literature. Second, it is recommended that-if applicable — the consent of both parents of the minor is required to avoid a lack of awareness in one of the parents. In the case of a minor who already understands the test and its consequences, their valid consent to being tested should form a part of the process. Finally, together with Phillips, ${ }^{25}$ we voice the need for more transparency in the DTC industry in general and call for a selfregulatory effort on behalf of industry leaders in the ancestry testing market in the development of concise and to the point minimum standards, which can serve as a code of conduct for DTC genetic ancestry testing companies.

\section{CONFLICT OF INTEREST}

The authors declare no conflict of interest.

\section{ACKNOWLEDGEMENTS}

MHDL is a postdoctoral fellow of the FWO-Vlaanderen (Research FoundationFlanders), and was supported by the KU Leuven BOF-C1-grant C12/15/013. We thank three anonymous reviewers, Anna Schaman, Stephanie Green and Kelly Nivelle for suggestions on a previous version of the manuscript.

1 Jobling MA, Hollox E, Hurles ME, Kivisild T, Tyler-Smith C: Human Evolutionary Genetics. London/New York: Garland Science Publishing, 2013.

2 Jobling MA, Rasteiro R, Wetton $\mathrm{JH}$ : In the blood: the myth and reality of genetic markers of identity. Ethn Racial Stud 2016; 39: 142-161.

3 Kirkpatrick BE, Rashkin MD: Ancestry testing and the practice of genetic counseling. J Genet Couns 26: 6-20.

4 Su P: Direct-to-consumer genetic testing: a comprehensive view. Yale J Biol Med 2013; 86: 359-365.

5 Royal CD, Novembre J, Fullerton SM et al: Inferring genetic ancestry: opportunities, challenges, and implications. Am J Hum Genet 2010; 86: 661-673.

6 Calafell F, Larmuseau MHD: The Y chromosome as the most popular marker in genetic genealogy benefits interdisciplinary research. Hum Genet 2016 (in press).

7 Via M, Ziv E, Burchard EG: Recent advances of genetic ancestry testing in biomedical research and direct to consumer testing. Clin Genet 2009; 76: 225-235.

8 Larmuseau MHD, Bekaert B, Baumers $\mathrm{M}$ et al: Biohistorical materials and contemporary privacy concerns-The forensic case of King Albert I. Forensic Sci Int Genet 2016; 24: 202-210.

9 Wallace SE, Gourna EG, Nikolova V, Sheehan NA: Family tree and ancestry inference: is there a need for a 'generational' consent? BMC Medical Ethics 2015; 16: 87.

10 Bolnick DA, Fullwiley D, Duster $T$ et al: The science and business of genetic ancestry testing. Science 2007; 318: 399-400.

11 Borry P, Rusu O, Dondorp W, De Wert G, Knoppers BM, Howard HC: Anonymity 2.0: direct-to-consumer genetic testing and donor conception. Fertil Steril 2014; 101: $630-632$.

12 Borry P, Rusu O, Howard HC: Genetic testing: anonymity of sperm donors under threat. Nature 2013; 496: 169 .

13 Lehmann-Haupt R: Are sperm donors really anonymous anymore? Slate 2010.

14 Harper JC, Kennett D, Reisel D: The end of donor anonymity: how genetic testing is likely to drive anonymous gamete donation out of business. Hum Reprod 2016; 31: 1135-1140.

15 Hudson K, Javitt G, Burke W, Byers P: ASHG statement* on direct-to-consumer genetic testing in the United States. Am J Hum Genet 2007; 81: 635-637.

16 Botkin JR, Belmont JW, Berg JS et al: Points to consider: ethical, legal, and psychosocial implications of genetic testing in children and adolescents. Am J Hum Genet 2015; 97: 6-21.

17 Davis DS: The changing face of 'misidentified paternity'. J Med Philos 2007; 32 : 359-373.

18 Gray PB, Anderson KG: Fatherhood-Evolution and Human Paternal Behavior. Cambridge (USA): Harvard University Press, 2010.

19 Juengst ET: Genetic testing and the moral dynamics of family life. Public Underst Sci 1999; 8: 193-205.

20 Howard HC, Knoppers BM, Borry P: Are the kids really all right? Direct-to-consumer genetic testing in children: are company policies clashing with professional norms? Eur J Hum Genet 2011; 19: 1122-1126.

21 Borry P, Howard HC, Senecal K, Avard D: Health-related direct-to-consumer genetic testing: a review of companies' policies with regard to genetic testing in minors. Fam Cancer 2010; 9: 51-59.

22 Anderson KG: How well does paternity confidence match actual paternity? Evidence from worldwide nonpaternity rates. Curr Anthropol 2006; 47: 513-520.

23 Larmuseau MHD, Matthijs K, Wenseleers T: Cuckolded fathers rare in human populations. Trends Ecol Evol 2016; 31: 327-329.

24 Larmuseau MHD, Matthijs K, Wenseleers T: Long-term trends in human extra-pair paternity: increased infidelity or adaptive strategy? A reply to Harris (2016). Trends Ecol Evol 2016; 31: 663-665.

25 Phillips AD: Only a click away-DTC genetics for ancestry, health, love...and more: a view of the business and regulatory landscape'. Appl Trans/ Genom 2016; 8: 16-22.

Supplementary Information accompanies this paper on European Journal of Human Genetics website (http://www.nature.com/ejhg) 